\title{
Metales pesados contenidos en los sedimentos de fondo y en la columna de agua del arroyo San Lorenzo, Departamento Central, Paraguay
}

\author{
Heavy metals contained in the bottom sediments and in the water \\ column of the San Lorenzo stream, Central Department, Paraguay \\ Néstor Salinas Franco ${ }^{1,2 *}$ (D) Julio Benitez ${ }^{1}$ (D) Tomás López ${ }^{1}$ \\ ${ }^{1}$ Universidad Nacional de Asunción, Facultad de Ciencias Exactas y \\ Naturales. San Lorenzo, Paraguay. \\ ${ }^{2}$ Concejo Nacional de Ciencia y Tecnología. Asunción, Paraguay.
}

Autor correspondiente: nestorsalinas@facen.una.py

Resumen: Se han estudiado tres tramos del cauce del arroyo San LorenzoParaguay, que en este trabajo se denominaron: Barcequillo, San Isidro y La Pradera, abarcando de esta manera la zona alta, media y baja de la cuenca del cauce hídrico. Se ha constatado la presencia de metales pesados tanto en el sedimento de fondo como en la columna de agua. En los sedimentos se ha determinado valores que superan los niveles máximos según normas de la NOAA-SQuiRTs de los E.E.U.U. para el Pb con $16 \mathrm{mg} / \mathrm{Kg}$ en La Pradera y para el $\mathrm{Hg}$ en los tres tramos, siendo $0,092 \mathrm{mg} / \mathrm{Kg}$ el valor máximo en el mismo tramo. En tanto que, en agua, los valores de los metales no han sobrepasado las normas de la SEAM - Res. $N^{\circ}$ 222/02. Finalmente, el Análisis de Componentes Principales de los parámetros fisicoquímicos en sedimento muestra relación de la materia orgánica, $\mathrm{pH}, \mathrm{K}^{+}, \mathrm{P}$ y la granulometría fina (arena fina y limo/arcilla) con metales como el $\mathrm{Fe}, \mathrm{Zn}$ y $\mathrm{Cr}$, en el punto de muestreo Barcequillo.

Palabras clave: metales pesados, sedimento-agua, Arroyo San Lorenzo.

Abstract: Three sections of the San Lorenzo stream channel have been studied, which in this work were called Barcequillo, San Isidro and La Pradera areas, trying to cover the upper, middle and lower areas of the basin of said channel. The presence of heavy metals has been verified both in the sediment and in the water column. In the sediments, high values have 
been determined according to the norms of the NOAA-SQuiRTs of the USA. in $\mathrm{Pb}$ of $16 \mathrm{mg} / \mathrm{Kg}$ in La Pradera and $\mathrm{Hg}$ in the three sections, with 0.092 $\mathrm{mg} / \mathrm{Kg}$ being the maximum value in the same section. While in water, the values of the metals under study have not exceeded the standards of SEAM Res. $N^{\circ} 222 / 02$. Finally, regarding the Principal Component Analysis of the physicochemical parameters in sediment, there was a verifiable relationship between Organic Matter, $\mathrm{pH}, \mathrm{K}^{+}, \mathrm{P}$ and fine grain size (fine sand and silt / clay) with metals such as $\mathrm{Fe}, \mathrm{Zn}$ and $\mathrm{Cr}$, at the Barcequillo sampling point.

Keywords: heavy metals, sediment-water, arroyo San Lorenzo.

\section{INTRODUCCIÓN}

El estudio de sedimentos que han sido erosionados, trasportados y depositados por los cauces hídricos es de gran importancia donde la granulométrica y principalmente las arcillas juegan un papel determinante en la acumulación de sustancias contaminantes como los metales pesados(1) por la capacidad de los sedimentos finos de absorber y desorber las mismas. La interacción entre la columna de agua y el sedimento del lecho en un cauce determinará el comportamiento de los contaminantes, por lo tanto, la permanencia de estas en suspensión posibilitaría una mayor dispersión hacia cursos hídricos mayores ${ }^{(1,2,3)}$

La calidad de los sedimentos de fondo de un lecho es determinada también por otros parámetros fisicoquímicos como el $\mathrm{pH}$, las condiciones redox, la presencia de óxidos e hidróxidos de Fe y Mn y la variación del contenido de materia orgánica definen la disponibilidad de los solutos para ser transferidos desde los sedimentos a la columna de agua(4). La evaluación de la calidad ambiental de los cursos hídricos, comúnmente es determinado solo en la columna de agua sin tener en cuenta los sedimentos del fondo del cauce(5). Los primeros estudios relacionado a metales pesados en sedimentos en el país, fue realizado por Facetti et al.(6), quienes estudiaron los sedimentos del lecho del rio Paraguay, a lo largo de todo su tramo. En general, las concentraciones disminuyen en el orden $\mathrm{Fe}>\mathrm{Mn}>\mathrm{Zn}>\mathrm{Cr}>\mathrm{Pb}>$ $\mathrm{Cu}$, siendo los valores más altos correspondientes a la cercanía de efluentes de cauces urbanos como ciertos arroyos de la capital. También Figueredo ${ }^{(7)}$, realizo un estudio pormenorizado de metales pesados en sedimentos y aguas del arroyo San Lorenzo (ASL) en el tramo que atraviesa el Campus Universitario de la Universidad Nacional de Asunción. En su estudio constato la alta presencia de $\mathrm{Cr}, \mathrm{Cd}$ y $\mathrm{Zn}$ en sedimentos por encima de la norma EPA de los EEUU. 
En la zona metropolitana de Asunción se encuentran varias microcuencas de alta importancia ambiental debido a que ocupan zonas urbanas y suburbanas. Las precipitaciones arrastran materiales de escorrentías y efluentes antropogénicas que en parte se infiltran en el suelo y luego al Acuífero Patiño, otra parte discurre por cauces hídricos de bajo caudal y de tramos cortos que depositan sus aguas y sedimentos directamente al Rio Paraguay o a la Cuenta del Lago Ypacaraí. En esta última se encuentra la Cuenca Hídrica del Arroyo San Lorenzo (CHASL) comprende un área de $68,08 \mathrm{~km}^{2}$, abarcando áreas urbanas densamente pobladas, y que, en sus alrededores concentra un tipo muy variado de actividades a las que están sujetos los habitantes y personas que circulan por la zona, tales como viviendas particulares, comercios, asentamientos, industrias, áreas recreativas, escuelas, universidades, clubes deportivos, iglesias y hospitales(8). En tal sentido, se propone en este trabajo como objetivo estudiar metales trazas presentes en la columna de agua y en los sedimentos de fondo del cauce.

\section{MATERIALES Y MÉTODOS}

Lugares de muestreo y técnicas de campo:

Se realizaron los muestreos de las aguas, luego de los sedimentos en tres tramos del ASL: en la cabecera, el tramo medio, hasta casi la desembocadura (Figura 1), teniendo en cuenta abarcar toda la longitud del cauce, con los tributarios menores que acarrean sedimentos de la CHASL (Tabla 1). Las muestras de sedimentos se colectaron por muestreo integrado ${ }^{(9,10)}$ que consiste en realizar varias tomas de muestras puntuales de cada sitio de muestreo y mezclar para poder evitar la variabilidad que puedan tener.

Tabla 1. Puntos de muestreo de sedimentos y de agua.

\begin{tabular}{|c|c|c|c|c|}
\hline \multirow{2}{*}{$\begin{array}{l}\text { Puntos de } \\
\text { muestreo }\end{array}$} & \multicolumn{2}{|c|}{ Coordenadas UTM $21 \mathrm{~J}$} & \multirow{2}{*}{$\begin{array}{l}\text { Cota en } \\
\text { m.s.n.m. }\end{array}$} & \multirow[t]{2}{*}{ Fecha } \\
\hline & $\mathrm{X}$ & $\mathrm{Y}$ & & \\
\hline Barcequillo & 445.828 & 7.196 .010 & 146 & $07 / 09 / 2018$ \\
\hline San Isidro & 449.397 & 7.198 .090 & 106 & $07 / 09 / 2018$ \\
\hline La Pradera & 454.222 & 7.200 .051 & 85 & $07 / 09 / 2018$ \\
\hline
\end{tabular}

Salinas Franco N, Benitez J, López T. Metales pesados contenidos en los sedimentos de fondo y en la columna de agua del arroyo San Lorenzo, Departamento Central, Paraguay 


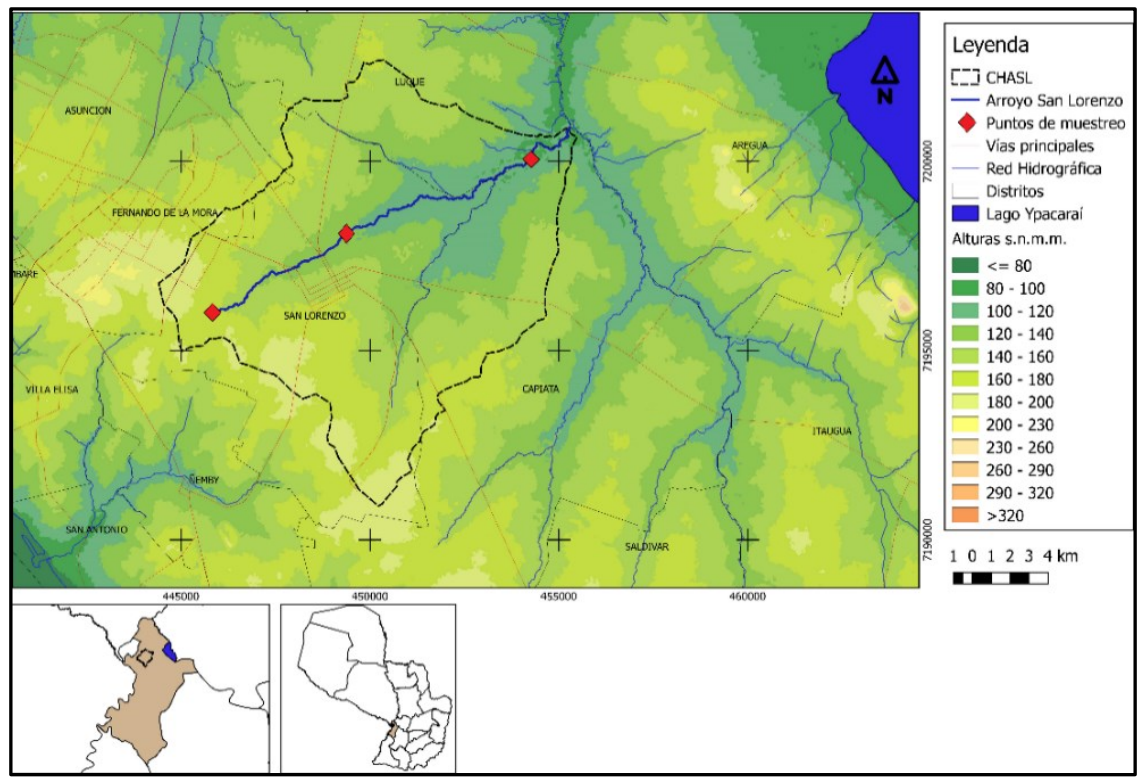

Figura 1. Mapa Altimétrico con la ubicación del área de estudio. Fuente: Propia.

Para la colecta de muestras de agua fueron utilizados bidones de polietileno con capacidad de 5 litros. Previamente los bidones eran enjuagados tres veces con el agua del lugar atendiendo a no perturbar el lecho. A las muestras de agua colectadas inmediatamente se les agregaban 5 gotas de ácido nítrico $\left(\mathrm{HNO}_{3}\right)$ concentrado y trasportados en conservadoras hasta el laboratorio. También se realizaron mediciones de parámetros fisicoquímicos in situ al agua de cada uno de los tramos de muestreo. Con el uso de un equipo Multiparamétrico se determinó el, oxígeno disuelto (OD en $\mathrm{mg} / \mathrm{L})$, temperatura $\left({ }^{\circ} \mathrm{C}\right)$, conductividad eléctrica (CE en $\mathrm{mS} / \mathrm{m}$ ), salinidad (UPS), los sólidos totales disueltos (TDS, calculado) y turbidez (NTU).

\section{Trabajos de Laboratorio}

Los análisis de metales como $\mathrm{Cr}$ (total), $\mathrm{Hg} \mathrm{y} \mathrm{Pb}^{(11)}$ tanto para agua como para sedimento fueron realizados en el Laboratorio de Calidad de Agua de la Facultad de Ciencias Exactas y Naturales (FACEN-UNA). Mientras que los restantes metales $\mathrm{Fe}, \mathrm{Mn}, \mathrm{Cu}$ y $\mathrm{Zn}^{(12)}$ fueron determinados para sedimentos en el Laboratorio del Departamento de Suelos y Ordenamiento Territorial de la Facultad de Ciencias Agrarias (FCA-UNA) (Tabla 2). 
Rev. Soc. cient. Parag. 2021;26(1):100-114

Tabla 2. Técnicas de laboratorio utilizadas para la determinación de metales pesados.

\begin{tabular}{|c|c|c|c|}
\hline \multirow[t]{2}{*}{ Elemento } & \multicolumn{2}{|c|}{ Método } & \multirow[t]{2}{*}{ Laboratorio } \\
\hline & Sedimento & Agua & \\
\hline Cr (total) & $\begin{array}{l}\text { (SM 3500-Cr D) APHA- } \\
\text { AWWA-WPCF, } 1992\end{array}$ & $\begin{array}{l}\text { (SM 3500-Cr D) } \\
\text { APHA-AWWA-WPCF, } \\
1992\end{array}$ & $\begin{array}{l}\text { LAB. AGUA } \\
\text { FACEN-UNA }\end{array}$ \\
\hline $\mathrm{Hg}$ & $\begin{array}{l}\text { (SM 3500-Hg) APHA- } \\
\text { AWWA-WPCF, } 1992\end{array}$ & $\begin{array}{l}\text { (SM 3500-Hg) APHA- } \\
\text { AWWA-WPCF, } 1992\end{array}$ & $\begin{array}{l}\text { LAB. AGUA } \\
\text { FACEN-UNA }\end{array}$ \\
\hline $\mathrm{Pb}$ & $\begin{array}{l}\text { (SM } 3030, \text { SM } 3500 \mathrm{~Pb} \\
\text { B) APHA-AWWA-WPCF, } \\
1992\end{array}$ & $\begin{array}{l}\text { (SM 3030, SM } 3500 \\
\text { Pb B) APHA-AWWA- } \\
\text { WPCF, } 1992\end{array}$ & $\begin{array}{l}\text { LAB. AGUA- } \\
\text { FACEN-UNA }\end{array}$ \\
\hline $\mathrm{Fe}$ & Mehlich 1 & - & $\begin{array}{l}\text { LAB. SUELO } \\
\text { FCA-UNA }\end{array}$ \\
\hline $\mathrm{Mn}$ & Mehlich 1 & - & $\begin{array}{l}\text { LAB. SUELO } \\
\text { FCA-UNA }\end{array}$ \\
\hline $\mathrm{Cu}$ & Mehlich 1 & - & $\begin{array}{l}\text { LAB. SUELO } \\
\text { FCA-UNA }\end{array}$ \\
\hline Zn & Mehlich 1 & - & $\begin{array}{l}\text { LAB. SUELO } \\
\text { FCA-UNA }\end{array}$ \\
\hline
\end{tabular}

En los sedimentos de fondo también se realizaron análisis de algunos parámetros fisicoquímicos como el $\mathrm{pH}$, materia orgánica (M.O), fosforo total (P), cationes mayoritarios como $\mathrm{Ca}^{+2}, \mathrm{Mg}^{+2}, \mathrm{~K}^{+}, \mathrm{Na}^{+}$y la acides intercambiable $\mathrm{Al}^{+3}+\mathrm{H}^{+}$. Por último, para la determinación de los tamaños del grano se realizó por el Método Tamizado en el Laboratorio de Sedimentología de la FACEN-UNA. Para cuantificar la proporción de Arena, Limo-Arcilla, se utilizaron un set de tamices de $400 \mu \mathrm{m}$ para arena gruesa, 350 y $250 \mu \mathrm{m}$ arena media, 125, 90 y $63 \mu \mathrm{m}$ para arena fina y en la base (limo-arcilla).

\section{Trabajos de Gabinete}

Con las lecturas de los valores de metales $\mathrm{Cr}, \mathrm{Hg}, \mathrm{Pb}, \mathrm{Cr}, \mathrm{Zn}, \mathrm{Mn}, \mathrm{Cu}$ y $\mathrm{Fe}$, parámetros fisicoquímicos y granulométricos, se procedió realizar gráficos de distribución y tablas por cada lugar de muestreo. Fueron contrastando la concentración de los metales pesados con normas y tenores de referencia nacionales para agua en base a la Res. 222/02-SEAM (13) e internacionales en base a la NOAA-SQuiRTs ${ }^{(14)}$ para la concentración de estos elementos en el sedimento. También se realizó un Análisis de Componentes Principales (ACP) para conocer las variables que explican el mayor porcentaje de variabilidad acumulada tanto para el comportamiento de variables en agua, 
así como, en sedimentos en referencia al lugar de muestreo. Estos análisis se realizaron con ayuda del programa InfoStat 2017v.1.2.

\section{RESULTADOS Y DISCUSIÓN}

En el comportamiento de los parámetros fisicoquímicos in situ (Tabla 3) existe una semejanza entre la Conductividad Eléctrica (CE), la Salinidad y los Solidos Totales Disueltos (TDS, por sus siglas en inglés) aumentando estos progresivamente desde la zona alta del ASL, hasta la zona cercana a la desembocadura (La Pradera). Esto se puede deber en parte a los cationes solubles principalmente del $\mathrm{Ca}^{+2}$, el $\mathrm{Mg}^{+2}$ y el $\mathrm{Na}^{+}$presentes en el sedimento de fondo, similares a las condiciones del agua superficial del ASL determinados por Alvarenga, D. et al.(15) atribuyéndoles a entornos de agua dulce bicarbonatadas calco-magnésicas, corroborados por las condiciones ligeramente alcalinas que presentan el agua en los tres tramos. Estos resultados, también coinciden con Figueredo ${ }^{(7)}$ en sus lecturas de $\mathrm{pH}$ en el agua, que determino el valor promedio de 7,37, 7,16, 8,77 y 7,05 en puntos cercanos a San Isidro en esta investigación. También la temperatura del agua tiene un comportamiento similar, pero esto se puede deber a que en el momento de la medición se realizaron en distintas horas del día, ya que la incidencia de la luz solar directamente lo determina (16) siendo Barcequillo medido a las 9:00 hs, San Isidro a las 13:00 hs. Estos dos lugares de muestreo presentaban alta cobertura forestal, y La Pradera medido a las 16:00 hs, no presenta cobertura forestal en el cauce muestreado. Por otra parte, si existe semejanza Temperatura-OD, disminuyendo esta última en donde existe mayor temperatura, presentado valores críticos para la biodiversidad acuática en San Isidro y La Pradera, aunque también la causa de esta disminución en la concentración de $\mathrm{O}_{2}$ se puede asociar a las descargas domesticas e industriales en los tramos determinados(17).

Tabla 3. Parámetros fisicoquímicos in situ medidos en el agua.

\begin{tabular}{|c|c|c|c|c|c|c|c|}
\hline Punto & $\mathbf{p H}$ & $\begin{array}{l}\text { OD en } \\
\mathrm{mg} / \mathrm{L}\end{array}$ & $\begin{array}{c}\text { CE } \\
\mu \mathrm{S} / \mathrm{cm}\end{array}$ & $\begin{array}{c}\text { Turbide } \\
\text { z NTU }\end{array}$ & $\begin{array}{l}\text { Temper } \\
\text { atura } \\
{ }^{\circ} \mathrm{C}\end{array}$ & $\begin{array}{l}\text { Salinidad } \\
\text { UPS }\end{array}$ & $\begin{array}{l}\text { TSD } \\
\text { (cal.) }\end{array}$ \\
\hline Barcequillo & 7,36 & 4,54 & 299 & 38,9 & 21,5 & 0,1 & 206,35 \\
\hline San Isidro & 7,17 & 1,75 & 369 & 9,8 & 23,1 & 0,1 & 251,85 \\
\hline La Pradera & 7,45 & 1,59 & 548 & 8 & 23,1 & 0,2 & 368,2 \\
\hline $\begin{array}{l}\text { Desviación } \\
\text { Estándar }\end{array}$ & 0,1429 & 1,658 & 128,41 & 17,34 & 0,92 & 0,057 & 83,46 \\
\hline
\end{tabular}

Salinas Franco N, Benitez J, López T. Metales pesados contenidos en los sedimentos de fondo y en la columna de agua del arroyo San Lorenzo, Departamento Central, Paraguay 
En los parámetros fisicoquímicos en sedimentos determinados (Tabla 4), los de $\mathrm{pH}$ en los tres lugares de muestreo arrojan condiciones acidas siendo la de San Isidro el valor más acido con un 4,7. La Materia Orgánica (M.O.), fue determinada arrojando valores muy bajos en los tres lugares de muestreo siendo Barcequillo el de mayor valor con un 0,43 \%. Se determinó también la concentración del elemento fosforo (P), teniendo en los tres lugares de muestreo valores similares y bajos, siendo Barcequillo el de mayor valor con un $4,772 \mathrm{mg} / \mathrm{Kg}$. En cuanto a los cationes mayoritarios $\left(\mathrm{Ca}^{+2}, \mathrm{Mg}^{+2}, \mathrm{Na}^{+}, \mathrm{K}^{+} \mathrm{y}\right.$ $\mathrm{Al}^{+3}+\mathrm{H}^{+}$) se puede observar la abundancia de $\mathrm{Ca}^{+2}$ con respecto al restos cuyo mayor valor fue de 235,3 mg/kg en La Pradera.

Tabla 4. Parámetros fisicoquímicos, $\mathrm{P}$ y Cationes Mayoritarios en sedimento de fondo.

\begin{tabular}{|c|c|c|c|c|}
\hline \multirow[t]{2}{*}{ Parámetro } & \multicolumn{3}{|c|}{ Punto } & \multirow{2}{*}{$\begin{array}{l}\text { Desviación } \\
\text { Estándar }\end{array}$} \\
\hline & Barcequillo & San Isidro & La Pradera & \\
\hline pH & 5,19 & 4,7 & 4,94 & 0,2450 \\
\hline M.0. \% & 0,43 & 0,32 & 0,21 & 0,11 \\
\hline $\mathbf{P} \mathrm{mg} / \mathrm{kg}$ & 4,772 & 3,798 & 4,091 & 0,499 \\
\hline $\mathrm{Ca}^{+2}$ & 196,4 & 137,3 & 235,3 & 49,345 \\
\hline $\mathrm{Mg}^{+2}$ & 63,6 & 69,3 & 75,1 & 5,75007 \\
\hline $\mathrm{mg} / \mathrm{kg}$ & 32,8 & 18 & 10,9 & 11,173 \\
\hline $\mathrm{Na}^{+}$ & 0 & 0 & 3,4 & 1,962 \\
\hline $\mathrm{Al}^{+3}+\mathrm{H}^{+}$ & 52,2 & 112,4 & 84,3 & 30,122 \\
\hline
\end{tabular}

En la determinación de la granulometría (Figura 2) en los sedimentos, tuvieron comportamientos similares en los puntos San Isidro y La Pradera. En estos sitios de muestreo la proporción de sedimentos fue decreciendo de arena más gruesa hacia arena más fina, en estos mismos puntos la proporción de limo/arcilla arrojaron valores muy ínfimos. En tanto, el punto de muestreo Barcequillo presento comportamiento distinto en las arenas ya que su proporción fue decreciendo desde las arenas más finas, más abundantes, hacia las arenas más gruesas, y solo en este lugar de muestreo se obtuvo una proporción considerable de finos (limo/arcilla) de 1,35 \%. 
Rev. Soc. cient. Parag. 2021;26(1):100-114

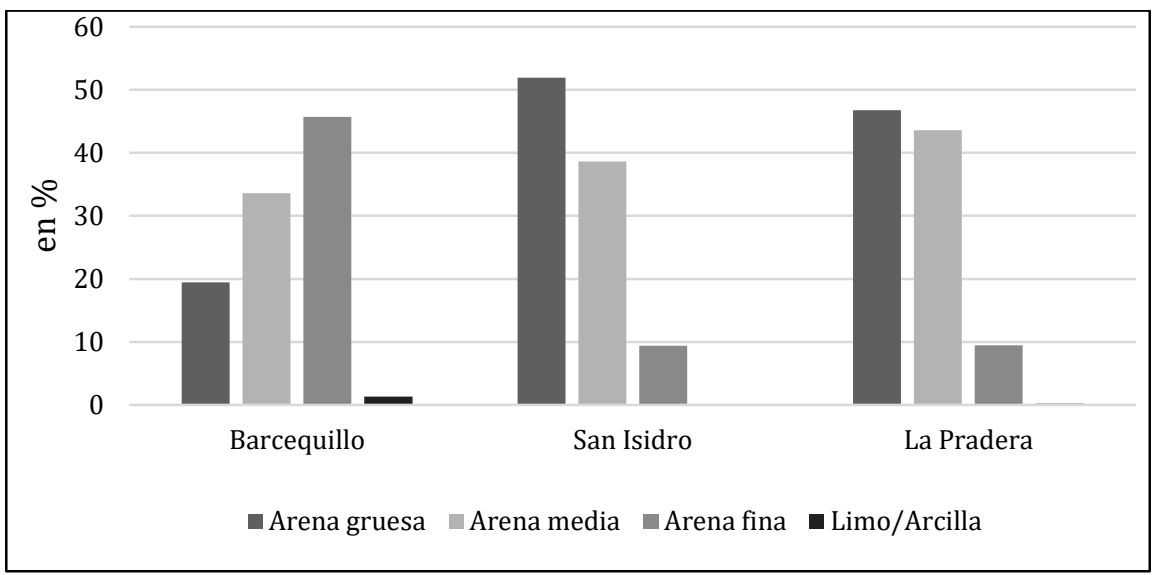

Figura 2. Distribución granulométrica de sedimentos en los tres tramos estudiados.

El $\mathrm{pH}$ es un condicionante importante en la disponibilidad de metales pesados en agua siendo los medios ácidos favorables para la misma $(17,18,19)$. En los lugares estudiados el agua presentó condiciones neutras a ligeramente alcalinas, en tanto, en los sedimentos se presentaron condiciones acidas en todos los puntos muestreados. Los valores fueron $5,19,4,7$ y 4,94 en coincidencia con muestras de agua de pozos del acuífero subyacente que habían sido determinados en investigaciones cuyo valor promedio fue de $5,09{ }^{(8)}$. Y es de suponer que existan trasferencia del lecho hacia el acuífero debido a las condiciones no confinantes de esta, y más aún en la CHASL donde se encuentran aflorantes rocas de este acuífero. El contenido de M.O. en sedimento coincide en su concentración (aunque sean pequeñas proporciones) con el contenido porcentual de los sedimentos finos (limo/arcilla), en el punto Barcequillo, también ambas son comparables con la Turbidez del agua que presenta en el mismo punto de muestreo valores altos muy cercanos a parámetros máximos permisibles de calidad ambiental. La concentración del catión $\mathrm{K}^{+}$que presenta valores más altos en el punto Barcequillo se puede interpretar que es absorbido principalmente por cargas negativas de la M.O. y las arcillas en estas(20).

Salinas Franco N, Benitez J, López T. Metales pesados contenidos en los sedimentos de fondo y en la columna de agua del arroyo San Lorenzo, Departamento Central, Paraguay 
Rev. Soc. cient. Parag. 2021;26(1):100-114

Tabla 5. Concentración de metales pesados en agua.

\begin{tabular}{llllll}
\hline $\begin{array}{l}\text { Parámetro } \\
\text { en mg/ L }\end{array}$ & Barcequillo & San Isidro & $\begin{array}{l}\text { La } \\
\text { Pradera }\end{array}$ & $\begin{array}{l}\text { Desviación } \\
\text { Estándar }\end{array}$ & $\begin{array}{l}\text { Res. } \\
\mathbf{2 2 2} / \mathbf{0 2}\end{array}$ \\
\cline { 2 - 5 } & & & $\mathbf{N}^{\circ}$ & \\
\hline Cr (total) & $<0,001$ & $<0,001$ & $<0,001$ & 0 & 0,5 \\
\hline $\mathbf{H g}$ & 0,00084 & 0,00112 & 0,00104 & 0,0001414 & 0,002 \\
\hline $\mathbf{P b}$ & $<0,001$ & $<0,001$ & $<0,001$ & 0 & 0,03 \\
\hline
\end{tabular}

Las concentraciones de metales pesados en agua no presentaron cambios en cada tramo estudiado presentando concentraciones sin ninguna variabilidad para el $\mathrm{Pb}$ y el $\mathrm{Cr}$, en tanto, el $\mathrm{Hg}$ presenta valores similares para los tres puntos de muestreo, también sus proporciones son mínimas y no sobrepasan las normas nacionales establecidas en los tres elementos estudiados en este medio, no obstante en los análisis de agua de pozos ${ }^{(8)}$ de la CHASL, existen muestras que sobrepasan el tenor nacional para la concentraciones en plomo.

Tabla 6. Concentración de metales pesados en sedimentos de fondo.

\begin{tabular}{|c|c|c|c|c|c|}
\hline \multirow{2}{*}{$\begin{array}{l}\text { Parámetro } \\
\text { en } \mathrm{mg} / \mathrm{kg}\end{array}$} & \multicolumn{3}{|c|}{ Punto } & \multirow{2}{*}{$\begin{array}{l}\text { Desviación } \\
\text { Estándar }\end{array}$} & \multirow{2}{*}{$\begin{array}{l}\text { NOAA- } \\
\text { SQuiRTs }\end{array}$} \\
\hline & Barcequillo & San Isidro & La Pradera & & \\
\hline Cr (total) & 2,47 & 2,21 & 2,32 & 0,1305 & 7 \\
\hline Hg & 0,09 & 0,084 & 0,092 & 0,0041 & 0,004 \\
\hline $\mathbf{P b}$ & $<0,333$ & $<0,333$ & 16 & 9,0453 & 4 \\
\hline $\mathrm{Cu}$ & 0,91 & 1,81 & 1,81 & 0,523 & 10 \\
\hline $\mathbf{Z n}$ & 1,88 & 0,47 & 1,41 & 0,716 & 7 \\
\hline $\mathbf{F e}$ & 28,98 & 19,32 & 19,32 & 5,578 & 9900 \\
\hline Mn & 40,17 & 38,25 & 40,17 & 1,104 & 400 \\
\hline
\end{tabular}

National Oceanic and Atmospheric Administration - Screening Quick Reference Tables (NOAA - SquiRTs).

Las concentraciones de metales pesados en sedimentos son elevadas en relación al agua, ya que se depositan o son absorbidos en el sedimento en determinados momentos, en estas sí presentan variabilidad siendo la más llamativa la de $\mathrm{Pb}$ con elevadas concentraciones en el punto La Pradera (16 
$\mathrm{mg} / \mathrm{kg}$ ) sobrepasando la norma de referencia de niveles de fondo de la NOAA-SQuiRTs. Esto se puede atribuir a las cercanías en el que el tramo estudiado se encuentra con una vía de trasporte vehicular, que potencialmente emitan residuos de este elemento y que estos se depositen en los sedimentos del lecho, corroborado en concentraciones de este elemento detectado en varias muestras de pozos dentro de la CHASL en la referencia(8).

La concentración de $\mathrm{Hg}$ (Tabla 6) también sobrepasan la norma de referencia que establece como concentración máxima $0,004 \mathrm{mg} / \mathrm{Kg}$, en los tres tramos estudiados, y también son valores superiores para los tenores nacionales en agua de ahí la importancia de los procesos de adsorcióndesorción para determinar el intercambio sedimento-agua de este metal. El Cr presenta valores por debajo de la norma de referencia utilizada en esta investigación y poca variación en los tres tramos, no obstante Figueredo(7), en el punto 4 cercano a San Isidro en su estudio había constatado elevadas concentraciones de este elemento siendo $16,225 \mathrm{~g} / \mathrm{kg}$ el valor más alto.

El $\mathrm{Cu}$ y el $\mathrm{Zn}$ presentan valores que no sobrepasan las normas background, pero al ser nutrientes esenciales en el organismo no presentaría alguna preocupación, aunque sus concentraciones no estén muy alegadas de la norma de referencia. En el caso del Fe y el Mn, con valores muy alegados de la norma de referencia utilizada en este estudio no presenta alguna anormalidad ya que también se consideran nutrientes esenciales en el suelo. Por otro lado, todos estos valores de metales en sedimentos de fondo se encuentran por debajo de las normas canadienses de la Guía Provisional de Calidad del Sedimento, de los cuales no se espera efectos adversos a la biota(21). Guía esta que fue adaptada por Flores et al.(22) en tenores normalizados para la Corteza Superior, eventualmente útil para estimar el nivel de efecto indeseable. 


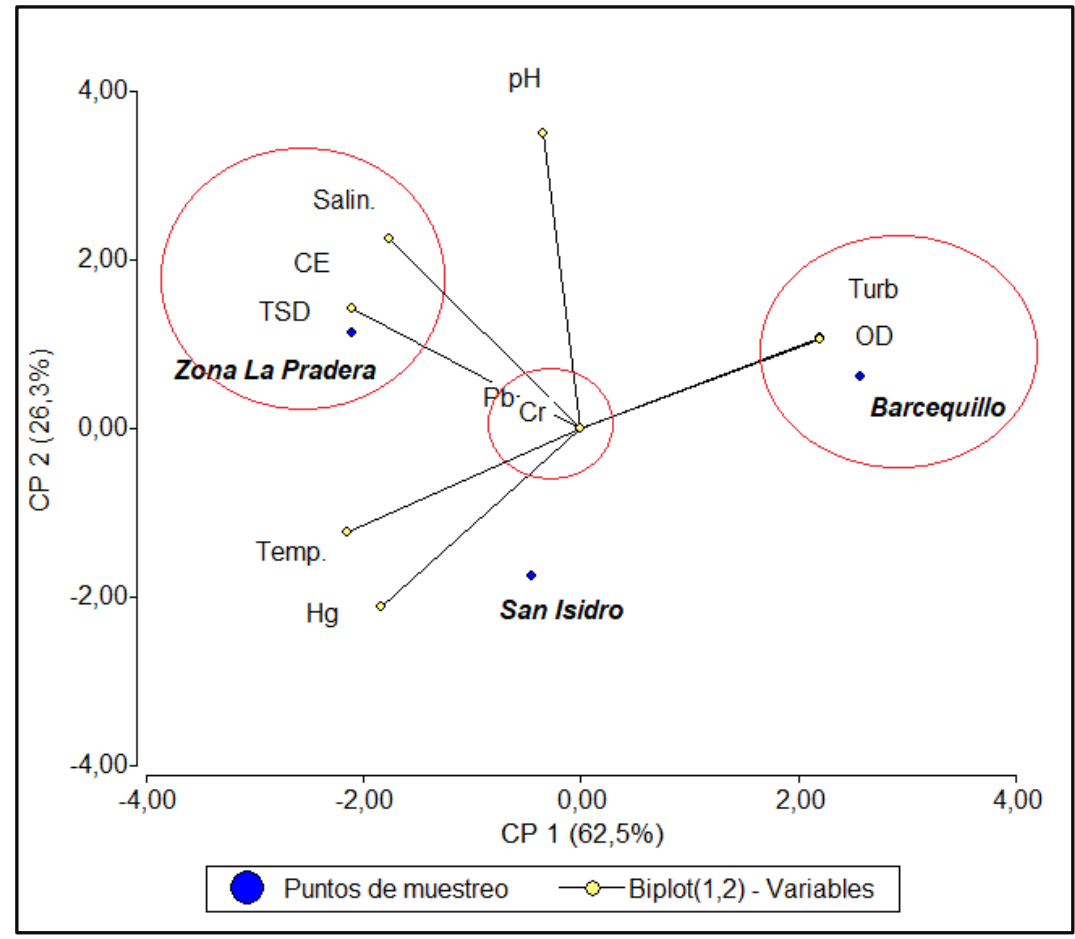

Figura 3. Análisis Componentes Principales determinado en agua.

En cuanto a los Análisis de Componentes Principales (ACP) realizado para variables del agua del ASL, fueron en base a parámetros fisicoquímicos como el $\mathrm{pH}$, conductividad eléctrica (CE), solidos totales disueltos (TSD), salinidad, turbidez, oxígeno disuelto (OD) y la temperatura, y también en base a las concentraciones de metales pesados como el $\mathrm{Cr}, \mathrm{Pb}$ y $\mathrm{Hg}$ en los tres tramos en estudio (Figura 3).

El primer componente (CP1) explica el 62,5 \% de la varianza, representando en ambas componentes el 88,8 \% de la variabilidad. En las correlaciones con las variables originales se ven aquellas $\mathrm{CP}$ que tienen correlaciones y lo ideal es que exista alta correlación de cada variable con una sola CP. La CP1 se correlacionó positivamente con OD y la turbidez, y negativamente con $\mathrm{CE}$, temperatura, salinidad, TSD y $\mathrm{Hg}$, en tanto la CP2 se correlacionó positivamente con el $\mathrm{pH}$ No existe variabilidad en las concentraciones de $\mathrm{Pb}$ y Cr para los tres puntos de muestreo. 
Rev. Soc. cient. Parag. 2021;26(1):100-114

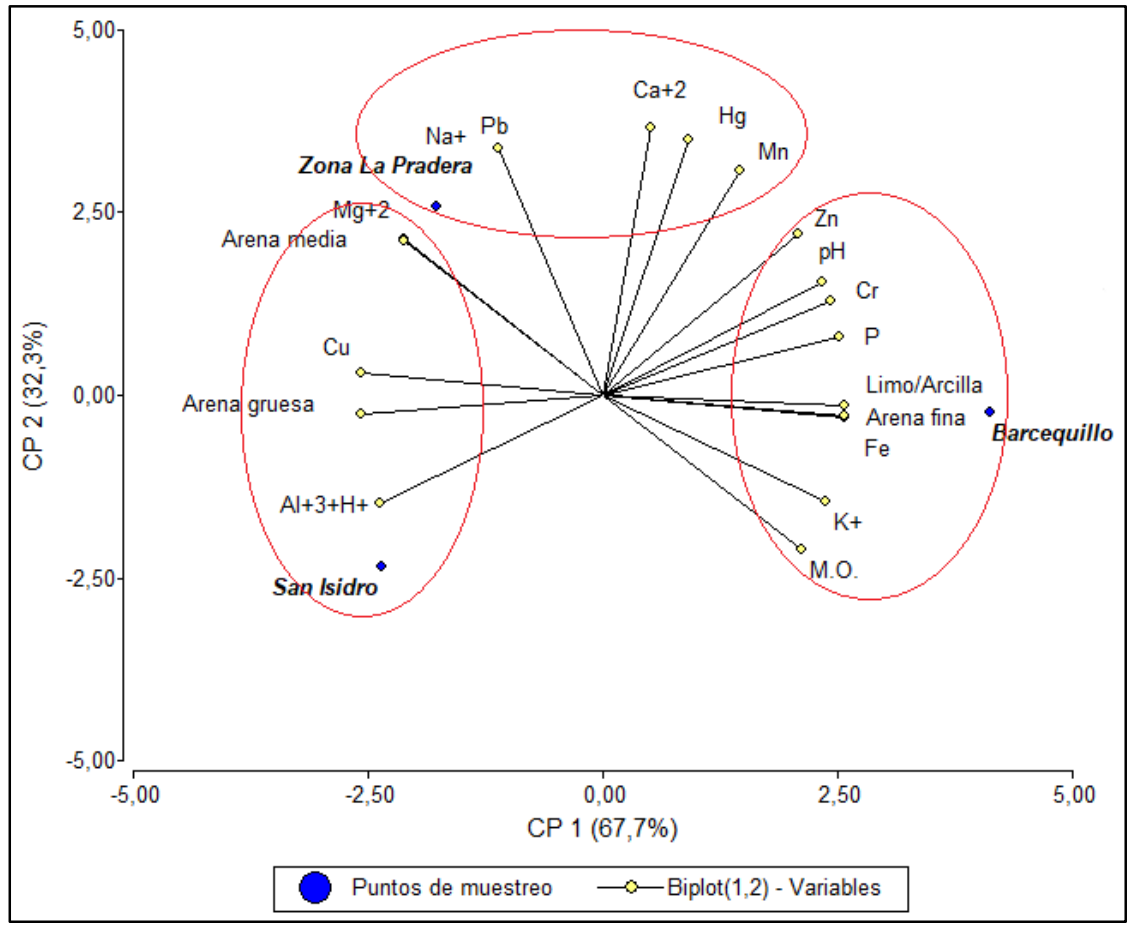

Figura 4. ACP determinado en sedimentos de fondo.

En sedimentos el primer Componente (CP1) explica el 67,7 \% de la varianza, y el CP2 explica el 32,3 representando en ambas componentes el $100 \%$ de la variabilidad. $\mathrm{La} \mathrm{CP} 1$ se correlacionó positivamente con $\mathrm{pH}, \mathrm{M} . \mathrm{O} ., \mathrm{P}, \mathrm{K}^{+}, \mathrm{Zn}$, $\mathrm{Fe}, \mathrm{Cr}$, Arena fina y Limo/Arcilla y negativamente con $\mathrm{Mg}^{+2}, \mathrm{Al}^{+3}+\mathrm{H}^{+}, \mathrm{Cu}$, arena gruesa y arena media, en tanto la $\mathrm{CP} 2$ se correlaciono positivamente con $\mathrm{Ca}^{+2}, \mathrm{Na}^{+}, \mathrm{Mn}, \mathrm{Hg}$ y $\mathrm{Pb}$ (Figura 4).

En los ACP tanto para el agua como para el sedimento se evidencia una relación contrastable entre los parámetros fisicoquímicos y las concentraciones de iones mayoritarios con los metales pesados, en donde para agua es menor esta relación, más aún porque $\mathrm{Pb}$ y $\mathrm{Cr}$ no existe variabilidad, recayendo en el $\mathrm{Hg}$ mayor variabilidad para el punto de muestreo San Isidro correlacionándose negativamente con la CP1. Para sedimentos con una amplia gama de variables introducidos para el ACP, existe una contrastable relación de parámetros fisicoquímicos como la M.O., $\mathrm{pH}, \mathrm{K}^{+}, \mathrm{P}$ y la granulometría fina (arena fina y limo/arcilla) con metales como el $\mathrm{Fe}$, $\mathrm{Zn}$ y Cr, en el punto de muestreo Barcequillo, correlacionándose positivamente estos parámetros al CP1. Cationes mayoritarios como el $\mathrm{Ca}^{+2}$ y el $\mathrm{Na}^{+}$están relacionados con metales pesados como el $\mathrm{Mn}, \mathrm{Hg}$ y

Salinas Franco N, Benitez J, López T. Metales pesados contenidos en los sedimentos de fondo y en la columna de agua del arroyo San Lorenzo, Departamento Central, Paraguay 
principalmente $\mathrm{Pb}$ en el punto de muestreo La Pradera, correlacionado positivamente con la CP2. En Contraste el punto de muestreo San Isidro presenta mayor acides potencial $\left(\mathrm{A}^{+3}+\mathrm{H}^{+}\right)$y concentración de arena gruesa (rico en $\mathrm{SiO}_{2}$ ) relacionándose con el metal $\mathrm{Cu}$, y estas últimas se correlacionan negativamente con la CP1.

\section{CONCLUSIONES}

Para la columna de agua del arroyo San Lorenzo en los sitios estudiados muestran que las concentraciones de los metales $\mathrm{Cr}, \mathrm{Hg}$ y $\mathrm{Pb}$, no sobrepasan los tenores establecidos por la legislación nacional (por Res. $N^{\circ} 222 / 02$ ), sin embargo, existe concentraciones cercanas a estas normas, para el $\mathrm{Pb}$ y Hg. Por otra parte, existe cierta relación entre los parámetros fisicoquímicos determinados en el agua y las concentraciones de iones mayoritarios presentes el sedimento.

El lecho del cauce en el punto Barcequillo se presenta prácticamente seco, debido a esto los sedimentos finos predominan en mayor medida (arena fina y mayor porcentaje limo/arcilla en comparación con los otros puntos estudiados) albergando la predominancia de $\mathrm{P}, \mathrm{K}^{+}$, y la M.O, asociados a metales como el Fe, Zn y Cr.

Se aprecian concentraciones notables de metales como el $\mathrm{Pb}$ y $\mathrm{Hg}$ en el sedimento que superan las normas de referencia internacional basada en niveles de fondo (NOAA-SQuiRTs), pero si usáramos las normas nacionales establecidas en tenores para agua (por Res. $\mathrm{N}^{\circ} 222 / 02$ ) estas superan ampliamente para los mismos metales estudiados en agua, es decir para el $\mathrm{Cr}, \mathrm{Hg}$ y $\mathrm{Pb}$. Con esto se deduce que dependerá de los procesos químicos de absorción-desorción para determinar el grado de intercambio entre en el sistema sedimento-agua de estos metales y su disponibilidad en la columna de agua en determinados momentos.

En los ACP tanto para agua y para sedimento, muestra relación de los parámetros fisicoquímicos, la granulometría y los metales en estudio, se puede notar esta relación más acentuada para los ACP realizados en sedimentos del lecho del arroyo. Siguiendo a (1) es importante cambiar los paradigmas en cuanto a los estudios de las condiciones ambientales tratando de abarcar todas las áreas de la ciencia (siendo la sedimentología no menos importante), en la interdisciplinariedad y el enfoque multisectorial. Esto ayudara a una mejor comprensión del medio natural y de los procesos que en ella actúan. 


\section{AGRADECIMIENTOS}

Al Programa PROCIENCIA del Concejo Nacional de Ciencia y Tecnología, Paraguay (CONACYT) y a la Facultad de Ciencias Exactas y Naturales - UNA, por la beca otorgada mediante la Maestría en Elaboración, Gestión y Evaluación de Proyectos de Investigación Científica

\section{REFERENCIAS BIBLIOGRÁFICAS}

1. Bidegain JC, Jurado S. Adsorción de contaminantes en sedimentos del Holoceno de la Región de la Plata. Revista Ciencia y Tecnología de los materiales. 2011;(1):75-84.

2. Hondzo M.Dissolved oxygen transfer at the sediment-water interface in a turbulent flow. Water Resources Res. 1998;34(12).

3. Steinberger N; Hondzo M. A semi-analytical model for dissolved oxygen mass transfer coefficient at the sediment-water interface. Journal. Hydraulic Engineering. 1999;125(2).

4. Huettel M, Webster IT. The Benthic Boundary Layer. Transport processes and biogeochemistry. Oxford University Press, 2001.

5. Molina PX, Rodríguez BX, Aránguiz AA. Análisis de la composición físico química de los sedimentos fluviales y su relación con la disponibilidad de metales en agua. Centro Nacional del Medio Ambiente (CENMA). Santiago, Chile; 2010, 69.

6. Facetti J, Dekov VM, Van Grieken R. Heavy metals in sediments from the Paraguay River: a preliminary study. Science of the total environment. 1998;209(1):79-86.

7. Figueredo G. Determinación de metales pesados en agua y sedimentos en el Arroyo San Lorenzo (tesis). Dirección General de Postgrado y Relaciones Internacionales. Universidad Nacional de Asunción; 2012.

8. Secretaría del Ambiente - SEAM. Proyecto "Manejo Sostenible y Protección de las Aguas Subterráneas en Paraguay" (PAS-PY). Investigación de la Calidad del Agua. Cuenca Hídrica del Arroyo San Lorenzo, Departamento Central Informe- BGR. Asunción; 2012.

9. Instituto de Hidrología, Meteorología y Estudios Ambientales-IDEAM. Protocolo Muestreo en aguas y sedimentos para metales. Ministerio de Ambiente, Vivienda y Desarrollo Territorial - República de Colombia; 2009.

10. Menares K, López A. Análisis de muestreos rutinarios e integrados de sedimentos en suspensión: caso de cuencas en Chile Central. XXV Congreso Latinoamericano de Hidráulica. San José, Costa Rica; 2012.

11. American Public Health Association. Métodos normalizados para el análisis de aguas potables y residuales. Ediciones Díaz de Santos; 1992. 
12. McKean S. Manual de análisis de suelos y tejido vegetal: una guía teórica y práctica de metodologías; 1993.

13. Secretaría del Ambiente, Res. $N^{\circ}$ 222/02. Por la cual se establece el padron de calidad de las aguas en el territorio nacional. Asunción; 2002.

14. Buchman MF. NOAA screening quick reference tables; 1999.

15. Alvarenga D, Jara S, Fariña SL, Larroza F, Cabral N. Estudios de los riesgos de contaminación en pozos profundos en el Campus de la Universidad Nacional de Asunción (UNA) Paraguay VIII Congreso Latinoamericano de Hidrología Subterránea Asunción, Paraguay; 2006.

16. Eyre B, Balls P. A Comparative Study of Nutrient Behavior along the Salinity Gradient of Tropical and Temperate Estuaries. Estuaries. 1999;22(2):313. doi:10.2307/1352987

17. Mello De Carvalho L. Distribución espacial de metales pesados en la Cuenca del Arroyo Carrasco y su relación con los usos de la cuenca asociada (Tesis). Universidad de la República; 2012.

18. Macías HPG. Determinación de metales pesados ( $\mathrm{Pb}, \mathrm{Cd}, \mathrm{Cr}$ ) en agua y sedimientos de la zona estuarina del río Tuxpan, Veracruz (Tesis) Universidad Veracruzana. México; 2015.

19. Mauro LN. Estudio de los procesos de adsorción-desorción de los metales $\mathrm{Cu}, \mathrm{Mn}, \mathrm{Pb}$ y $\mathrm{Zn}$ en la cuenca del río Maipo (Tesis). Universidad de Chile, Santiago, Chile; 2014.

20.Turner A, Millward GE, Le Roux SM. Significance of oxides and particulate organic matter in controlling trace metal partitioning in a contaminated estuary. Marine Chemistry. 2014;88(3-4):179-192. doi:10.1016/j.marchem.2004.03.008

21. Canadian Council of Ministers of The Environment. Canadian sediment quality guidelines for the protection of aquatic life: Introduction. Updated. In: Canadian environmental quality guidelines, 1999, Canadian Council of Ministers of the Environment:Winnipeg; 2001.

22. Flores FD, Delgado M, Facetti JF. Sedimentos de fondo en los arroyos Tapiracuai y Cuarepotí. Revista de la Sociedad Científica del Paraguay. 2018;23(2):251-262. 